\title{
Wavelength Dispersive Spectroscopy analysis of GSR: a novel approach to firearm identification
}

\author{
M.K. Garren*, S.J. Singletary *, ** \\ * Dept. of Natural Sciences, Fayetteville State University, 1200 Murchison Road, Fayetteville, NC \\ 28301 \\ ** Southeastern North Carolina Regional Microanalytical and Imaging Center, 1200 Murchison \\ Road, Fayetteville, NC 28301
}

Gunshot residue (GSR) is generated whenever a firearm is discharged. GSR may contain parts of the shell casing, primer residue, pieces of the projectile as well as particles from the firearm itself. GSR is distributed widely around the weapon and may be found on the hands of the person that fired the weapon, their clothing, on people or objects near the firearm at the time of discharge and objects in the vicinity of the discharged weapon. Primer residue is the most abundant component of GSR and it is composed predominately of lead $(\mathrm{Pb})$, barium $(\mathrm{Ba})$ and antimony $(\mathrm{Sb})$ making these three elements the most common in GSR. SEM imaging and analysis of GSR has become a routine forensic tool. Investigators have used GSR to identify suspects that may have recently discharged a firearm, to locate an area in which a firearm was discharged, and recently, to provide identification of specific primer residues. The analysis of GSR has been expanded to include the detection and characterization of explosive particles. GSR and explosive particles may be recovered from a persons hands/clothing or the area in which a firearm was recently discharged by several different methods. Tape lift, vacuum lift and swabbing are two commonly used methods of GSR collection. The tape lift method is the most widely used because of the simplicity of the method. Once collected, the samples are then analyzed with an SEM that can provide images for comparison in order to match targets to shooters and suspects to weapons. SEM/Energy Dispersive Spectroscopy (EDS) analysis can also provide an elemental profile of GSR (Figure 1) although the analysis is qualitative. The ultimate goal of this work is to determine if Wavelength Dispersive Spectroscopy (WDS) can be used to quantitatively analyze the fraction of GSR particles from the firearm itself. The high resolution $(\sim 3 \mathrm{~nm})$ and small WDS spot size $(\sim 40 \mathrm{~nm}$ at $10 \mathrm{kV}, 10 \mathrm{nA})$ of the new JXA 8530F Electron Hyperprobe should allow for the classification and analysis of the majority of particles present in GSR. If successful, GSR from a variety of firearms of different manufacturers will be collected to determine if any variability in composition of the firearm particles can be detected. If so, this new analysis technique could provide Law Enforcement with a powerful new forensic tool.

Here we present the preliminary results of the application of the SENCR-MIC JEOL JXA$8530 \mathrm{~F}$ Hyperprobe to the analysis of GSR, with the specific aim of detecting, analyzing and characterizing particles of the weapon that was discharged. The goal is to be able to examine GSR and identify the manufacturer of the weapon based upon a metallurgical match. This has not been done previously due to a variety of reasons such as the vanishingly small percentage of weapon particles in comparison to primer residue; lack of instrumental precision and accuracy and lack of a database of weapon compositions. GSR particles were collected using the tape lift method with standard GSR recovery kits provided by the NC State Bureau of Investigation. Samples were collected from the hands and weapon of a test shooter that fired a Smith \& Wesson M\&P 9mm handgun on an indoor firing range. The GSR samples were then analyzed to determine the percentage of each particle type present (i.e. primer residue, propellant, projectile, etc.). The differentiation of each type of particle was made via EDS analysis (Figure 2). The overall percentage of weapon particles is reported and evaluated to determine if the proposed method of firearm identification is viable. 
Figure 1. Typical GSR particle, composed predominantly of $\mathrm{Pb}, \mathrm{Sb}$ and $\mathrm{Ba}$ (x4000 magnification)



Figure 2. EDS elemental profile of suspected projectile particle $(\mathrm{x} 18,000)$ magnification.

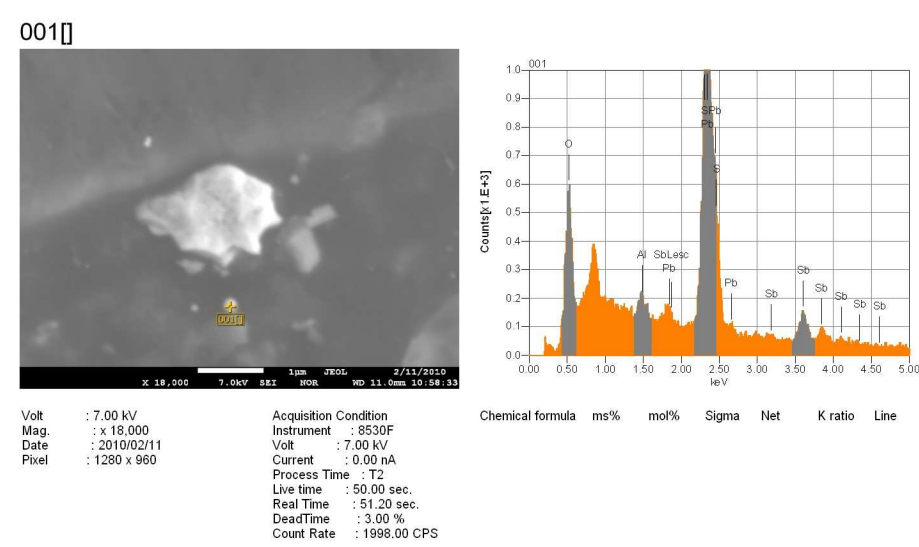

